\title{
EFECTO GASTROPROTECTOR DE Clinopodium bolivianum (BENTH.) KUNTZE “INCA MUÑA” SOBRE LESIONES GÁSTRICAS INDUCIDAS EN RATONES
}

\author{
Gastroprotector effect from Clinopodium bolivianum (Benth.) Kuntze "inca muña" on induced \\ gastric injury in mice \\ Oscar Herrera $^{1}$, Fanny Ventura ${ }^{1}$, Astrid Rivera ${ }^{1}$, Rita Valenzuela ${ }^{1}$, Martín Condorhuamán ${ }^{2}$ \\ ${ }^{1}$ Facultad de Farmacia y Bioquímica, Universidad Nacional San Luis Gonzaga. ${ }^{2}$ Instituto de Investigación en Ciencias Farmacéuticas \\ y Recursos Naturales "Juan de Dios Guevara", Facultad de Farmacia y Bioquímica, Universidad Nacional Mayor de San Marcos
}

\section{RESUMEN}

El objetivo del presente estudio fue determinar el efecto gastroprotector del extracto etanólico de Clinopodium bolivianum "inca muña" en lesiones gástricas inducidas en ratones. Se utilizaron 36 ratones albinos machos de la cepa Balb/C, de peso entre $30-35 \mathrm{~g}$, previamente mantenidos en ayuno $24 \mathrm{~h}$, solo con agua ad libitum. Las lesiones gástricas fueron inducidas con indometacina $2,5 \mathrm{mg} / \mathrm{Kg}$ (s.c.) una hora antes de administrar el extracto, complementando luego de 20 minutos con etanol $96 \%$, o, $1 \mathrm{~mL}$ por vía oral. El diseño experimental estuvo conformado por: G1 control negativo; $\mathrm{G} 2$ control positivo; $\mathrm{G}_{3}$ ranitidina $50 \mathrm{mg} / \mathrm{Kg} ; \mathrm{G}_{4}$, G5 y G6 extracto de inca muña a dosis de 50, 150 y $300 \mathrm{mg} / \mathrm{kg}$, respectivamente. Una hora después se extrajeron los estómagos y se evaluaron signos lesivos como: inflamación, bandas hemorrágicas y úlcera gástrica, y luego se realizaron estudios histológicos. El extracto etanólico de "inca muña" evidenció metabolitos secundarios como compuestos fenólicos, flavonoides, triterpenos y alcaloides y presentó efecto gastroprotector a la dosis de $300 \mathrm{mg} / \mathrm{Kg}$ en comparación al grupo control positivo (U- Mann Whitney; $p<0,001)$. En el estudio histológico se evidenció estómago con empastamiento en ápices e infiltración a mononucleares en base. Se concluye que el extracto etanólico de Clinopodium bolivianum tiene actividad gastroprotectora a la dosis de $300 \mathrm{mg} / \mathrm{Kg}$ sobre lesiones gástricas inducidas en ratones.

Palabras clave: Clinopodium, gastroprotector, lesión gástrica, indometacina, etanol.

\section{SUMMARY}

The objective of the study was to determine the effect gastroprotector of ethanolic extract of Clinopodium bolivianum on gastric injuries induced in mice, were used 36 albino male mice Balb/C, between $30-35 \mathrm{~g}$, previously fasted for $24 \mathrm{~h}$, only with water ad libitum. The gastric were injuries induced with indomethacin $2,5 \mathrm{mg} / \mathrm{Kg}$ (s.c.) one hour before administering the extract, complementing 20 minutes after with ethanol 96\%, o,1 mL orally. The experimental design was composed by: G1 negative control; G2 positive control; G3 ranitidine 50 mg Kg; G4, G5 y G6 extract 50, 150 y $300 \mathrm{mg} / \mathrm{kg}$, respectively. An hour later, the stomachs were extracted and evaluated as harmful signs: inflammation, hemorrhagic bands and gastric ulcer; and then histological studies were performed. In the results Inca muña ethanolic extract showed secondary metabolites as phenolic compounds, flavonoids, triterpenos and alkaloids. It was got gastroprotector effect to the $300 \mathrm{mg} / \mathrm{Kg}$ dose comparable to positive control group (U Mann Whitney; $p<0,001$ ). The stomach histology evidenced by pasting in apexes and mononuclear infiltration. Was concluded that ethanolic extract of Clinopodium bolivianum presents gastroprotector activity at $300 \mathrm{mg} / \mathrm{Kg}$ on gastric injuries induced in mice.

Keywords: Clinopodium, gastroprotector, gastric injury, indometacine, ethanol.

\section{INTRODUCCIÓN}

a úlcera péptica es una alteración de la mucosa gástrica y duodenal de carácter crónico y recurrente causada por la secreción ácida del estómago; es considerada un problema global con tasas de morbilidad y mortalidad en aumento ${ }^{(1)}$. Un desbalance entre los factores protectores (secreción de moco y bicarbonato, incremento de los niveles de antioxidantes y producción de prostaglandinas) y agresores de la mucosa (estrés, exposición a etanol y humo de tabaco, consumo frecuente de antiinflamatorios no esteroides), han sido asociados a la etiología de la enfermedad, así como la presencia del Helicobacter pylori en la mucosa gástrica y duodenal ${ }^{(2)}$.

Lasplantasaromáticassonunafuentenaturaldediversos antioxidantes. La familia Lamiaceae ha sido estudiada debido a su potencial actividad antioxidante, antiinflamatoria, antibacterial, antifúngica y propiedades antivirales. Se ha investigado la presencia de metabolitos secundarios en esta familia, tales como terpenoides, flavonoides (glicósidos y agliconas) y ácidos hidroxibenzóico e hidroxicinámicos ${ }^{(3,4)}$. Inca muña, conocida como el orégano de los incas o cjuñuca, es una planta salvaje aromática que pertenece a la familia Lamiaceae, crece en los andes centrales y sur del Perú (5). La medicina tradicional utiliza las hojas en infusiones para tratar la indigestión, nauseas, diarrea, anemia y enfermedades respiratorias; las flores y hojas han sido utilizadas como condimentos en diferentes preparados, sopas y dulces ${ }^{(5,6)}$; por todo ello, el objetivo de esta investigación fue evaluar el efecto gastroprotector del extracto etanólico de Clinopodium bolivianum (Benth.) Kuntze "inca muña” sobre las lesiones gástricas inducidas en ratones.

\section{MATERIAL Y MÉTODOS}

La investigación fue de tipo experimental, descriptivo y transversal. La especie vegetal fue colectada en el distrito de Paico, provincia de Sucre, región Ayacucho, a 3117 metros de altitud, en el mes de febrero, realizándose la clasificación taxonómica en el Museo de Historia Natural de la Universidad Nacional Mayor de San Marcos. 
El estudio farmacológico se realizó en el bioterio de la Facultad de Medicina "San Fernando" de la Universidad Nacional Mayor de San Marcos. Se emplearon ratones albinos Balb/C, adquiridos al bioterio del Instituto Nacional de Salud, aclimatados 14 días previos a la evaluación farmacológica a una temperatura de 18 a $22^{\circ} \mathrm{C}$ y ciclos de 12 horas luz/oscuridad. La alimentación de los animales de experimentación consistió en alimento balanceado y agua ad libitum.

\section{Obtención del extracto etanólico}

Constó de las siguientes etapas:

Selección y trituración: se seleccionaron las mejores partes de la planta para asegurar la calidad del extracto, obteniéndose triturado de planta entera.

Maceración: se colocaron 500 g del triturado en un frasco ambar de $3 \mathrm{~L}$ de capacidad, se le agregaron $2000 \mathrm{~mL}$ de etanol 96\%, y se cerró herméticamente por 7 días. Cada 24 horas se agitó el frasco por 5 minutos para maximizar el contacto de la planta triturada con el etanol y así asegurar la uniformidad y eficacia de la maceración.

Filtración, obtención del extracto y conservación: el macerado obtenido se filtró y el filtrado se llevó a la estufa a $40^{\circ} \mathrm{C}$, para evaporar el etanol, obteniéndose $15 \mathrm{~g}$ de extracto, el cual se refrigeró a $8^{\circ} \mathrm{C}$ en un frasco de color ámbar para su conservación.

Análisis fitoquímico. Se realizó mediante pruebas fisicoquímicas de caracterización, por los cambios de coloración o formación de precipitados ${ }^{(7)}$.

Evaluación del efecto gastroprotector. La inducción de úlcera gástrica fue realizada con indometacina y etanol de acuerdo al método propuesto por Robert et al (8). Posteriormente, para la etapa de sacrificio, se usó como anestésico tiopental sódico a una dosis de 100 $\mathrm{mg} / \mathrm{kg}$ de peso corporal.

Diseño experimental. Los ratones, divididos en 6 grupos, con 6 animales cada uno, fueron distribuídos de la siguiente manera: G1, control negativo; G2, control positivo; $\mathrm{G}_{3}$, ranitidina $50 \mathrm{mg} / \mathrm{kg} ; \mathrm{G}_{4}, \mathrm{G}_{5}, \mathrm{G} 6$, extractos a dosis de 50 , $150,300 \mathrm{mg} / \mathrm{kg}$. Fueron tratados con $2,5 \mathrm{mg} /$

Kg de indometacina por vía subcutánea, con excepción del grupo 1, una hora antes de la administración oral del extracto etanólico de Clinopodium bolivianum (vía oral). Los animales fueron sacrificados una hora después de recibir el extracto, extrayéndose los estómagos para el análisis de las lesiones. Estos fueron conservados en formaldehido al $40 \%$ tamponado en sodio fosfato monobásico monohidratado $(4 \%)$, sodio fosfato dibásico anhidro $(6,5 \%)$ con el fin de realizar la evaluación anatomopatológica.

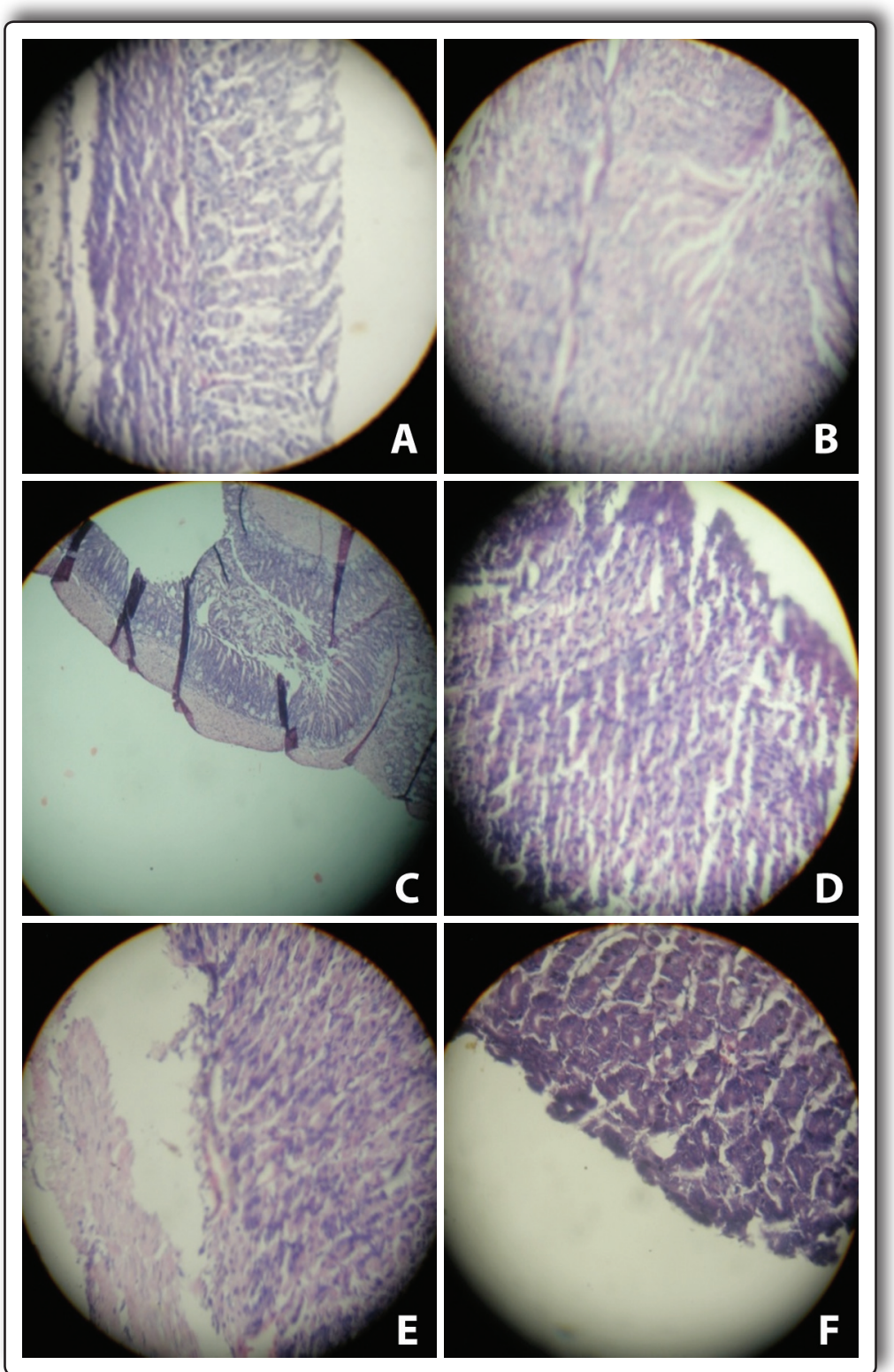

Figura 1. Microfotografía de estómagos en el tratamiento de lesiones gástricas. A (control negativo): estómago normal. B (control positivo): hipertrofia, luz con detritus, discreta erosión de mucosa, ápices deflecados, infiltración de mononucleares en tercio inferior (gastritis). C (Ranitidina $50 \mathrm{mg} / \mathrm{Kg}$ ); hipertrofia, luz con detritus, sin gastritis erosiva. D (Extracto $50 \mathrm{mg} / \mathrm{Kg}$ ): Infiltracion de células inflamatorias de ápex en la base. E (Extracto $150 \mathrm{mg} / \mathrm{Kg}$ ): discreta descamación superficial, edema de con empastamiento de ápices, infiltración a mononucleares en base.

Análisis estadístico. Luego de la ejecución del diseño experimental, los datos fueron ordenados y analizados, se aplicó el test de KrusKal Wallis con un nivel de significancia y U de Mann Whitney. Se consideró estadísticamente significativo para un $p<0,05$.

\section{RESULTADOS}

Los resultados obtenidos en el presente estudio se muestran en las tablas 1-4 y en la figura 1. 


\section{DISCUSIÓN}

El presente trabajo de investigación tuvo como objetivo evaluar el efecto gastroprotector del extracto etanólico Clinopodium bolivianum (Benth.) Kuntze "inca muña" sobre lesiones ulcerosas inducidas en ratones con indometacina y etanol -los que fueron escogidos basándose en lo descrito por Vonkeman et al ${ }^{(13)}{ }_{-}$. El estudio fitoquímico del extracto etanólico de toda la planta determinó que posee metabolitos como alcaloides, flavonoides y compuestos fenólicos (tabla 1). Tanto por el uso que se le dio durante el estudio, como en el uso popular, no se podría precisar si estos principios activos están en mayor porcentaje en alguna parte específica de la planta dado que se utiliza toda la especie. El resultado del daño causado podría deberse en parte a la formación de radicales libres, el etanol es metabolizado en el organismo causando un incremento de $\mathrm{O}_{2}^{-}$en los tejidos, causando daño directo al ADN y desnaturalización de proteínas ${ }^{(14)}$. En el estudio histológico se encontró que el daño causado a la mucosa gástrica por el etanol e indometacina, es caracterizado por edema y congestión de la mucosa ${ }^{(15)}$, observándose hipertrofia, luz con detritus, discreta erosión de mucosa, e infiltración de mononucleares en tercio inferior, en el grupo control positivo.

Los metabolitos secundarios con propiedad antioxidante de Clinopodium bolivianum actuarían como atrapadores de radicales libres, por lo que se podría asumir que entre ellos están los flavonoides. Los radicales libres juegan un rol en la depleción de los niveles de prostaglandinas, que inhiben la secreción de ácido y estimulan la secreción de moco y bicarbonato. Es así que las prostaglandinas contribuyen a proteger el estómago del daño producido por etanol; por ello, la citoprotección gástrica de Clinopodium bolivianum contra el daño producido se debería a una posible acción antioxidante.

En el presente estudio, la ranitidina no protege la mucosa gástrica del daño producido por etanol e indometacina; estudios reportados por Cadirci (16) y Mota (17), confirman este hallazgo. La indometacina disminuye la resistencia de la barrera gastrointestinal al etanol y reduce la secreción de prostaglandinas, por lo que el extracto podría proteger de los riesgos por esta disminución.

Por lo expuesto, es necesario mencionar que el aporte científico obtenido como resultado del presente estudio dará a la población mayor seguridad en el uso de esta especie vegetal como medicina tradicional $y$, asimismo, queda abierta la posibilidad para la industria de las plantas medicinales de establecer su composición química y elaborar una presentación farmacéutica que garantice su uso terapéutico.
Tabla 1. Análisis fitoquímico del extracto etanólico de Clinopodium bolivianum.

\begin{tabular}{lcc}
\hline Prueba de caracterización & Resultado & $\begin{array}{c}\text { Metabolito } \\
\text { secundario }\end{array}$ \\
\hline Reacción de la gelatina & $(-)$ & Taninos \\
Reacción con cloruro férrico & $(+)$ & Compuestos \\
Renólicos \\
Reacción de Dragendörff & $(+)$ & Alcaloides \\
Reacción Mayer & $(+)$ & Alcaloides \\
Reacción de Shinoda & $(+)$ & Alcaloides \\
Reacción de Borntrager & $(+)$ & Flavonoides \\
Reacción de & $(-)$ & Quinonas \\
Lieberman-Burchard & $(+)$ & Triterpeno y \\
Prueba de la espuma & $(-)$ & esteroides \\
\hline
\end{tabular}

(+) Presencia, (-) ausencia.

Tabla 2. Evaluación de la inflamación en el estómago de ratón en un modelo experimental de úlcera gástrica inducida por indometacina y etanol.

\begin{tabular}{|c|c|c|c|c|c|}
\hline \multirow{2}{*}{ Grupo experimental * } & \multirow{2}{*}{$\begin{array}{c}\text { Dosis } \\
\text { (mg/Kg) }\end{array}$} & \multicolumn{4}{|c|}{ Inflamación } \\
\hline & & $\mathrm{X} \pm \mathrm{DE}$ & Med & $\mathrm{p} 1$ & $\mathrm{p} 2$ \\
\hline Control negativo & - & $0,17 \pm 0,17$ & 0 & 0,005 & - \\
\hline Control positivo & - & $1,67 \pm 0,33$ & 1,5 & - & 0,005 \\
\hline IND/etanol + extracto inca muña & 50 & $0,83 \pm 0,17$ & 1 & 0,045 & 0,027 \\
\hline IND/etanol + extracto inca muña & 150 & $0,33 \pm 0,21$ & 0 & 0,011 & 0,523 \\
\hline IND/etanol + extracto inca muña & 300 & $0,17 \pm 0,17$ & 0 & 0,005 & 1 \\
\hline IND/etanol + ranitidina & 50 & $0,33 \pm 0,21$ & 0 & 0,011 & 0,523 \\
\hline $\begin{array}{l}\text { Los datos de ninguno de lo } \\
\text { olmogorov-Simirnov) X: media; } \\
\text { dometacina, p1: comparación en } \\
\text { indometacina } 2,5 \mathrm{mg} / \mathrm{kg} \text {, (Prue } \\
\text { hedianas de las dosis del extract }\end{array}$ & 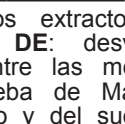 & $\begin{array}{l}\text { presentó } \\
\text { ación están } \\
\text { lianas de } \\
\text { n-Whitney); }\end{array}$ & $\mathrm{Cc}$ & normal & $\begin{array}{l}(p<0,0 \\
\text { a; INI } \\
\text { to y y } \\
\text { entre la }\end{array}$ \\
\hline
\end{tabular}

Tabla 3. Evaluación de bandas hemorrágicas en el estómago de ratón en un modelo experimental de úlcera gástrica inducida por indometacina y etanol.

\begin{tabular}{lccccc}
\hline \multirow{2}{*}{ Grupo experimental * } & Dosis & \multicolumn{3}{c}{ Bandas hemorrágicas } \\
\cline { 3 - 6 } & $(\mathrm{mg} / \mathrm{Kg})$ & $\mathbf{X} \pm \mathrm{DE}$ & Med & $\mathrm{p} 1$ & $\mathrm{p} 2$ \\
\hline Control negativo & - & $0,17 \pm 0,17$ & 0 & 0,003 & - \\
Control positivo & - & $2,33 \pm 0,42$ & 2 & - & 0,003 \\
IND/etanol + extracto inca muña & 50 & $1,67 \pm 0,21$ & 2 & 0,207 & 0,004 \\
IND/etanol + extracto inca muña & 150 & $0,83 \pm 0,48$ & 0,5 & 0,040 & 0,211 \\
IND/etanol + extracto inca muña & 300 & $0,33 \pm 0,21$ & 0 & 0,005 & 0,523 \\
IND/etanol + ranitidina & 50 & $0,33 \pm 0,21$ & 0 & 0,005 & 0,523 \\
\hline
\end{tabular}

* Los datos de ninguno de los extractos presentó distribución normal $(p<0,05$ Kolmogorov-Simirnov) X: media; DE: desviación estándar; Med: mediana; IND: indometacina, p1: comparación entre las medianas de las dosis del extracto y de la indometacina $2,5 \mathrm{mg} / \mathrm{kg}$, (Prueba de Mann-Whitney); p2: comparación entre las medianas de las dosis del extracto y del suero fisiológico, (Prueba de Mann-Whitney).

Tabla 4. Evaluación de úlcera gástrica en el estómago de ratón en un modelo experimental de úlcera gástrica inducida por indometacina y etanol.

\begin{tabular}{lccccc}
\hline \multirow{2}{*}{ Grupo experimental * } & Dosis & \multicolumn{4}{c}{ Ulcera gástrica } \\
\cline { 3 - 6 } & $(\mathbf{m g} / \mathbf{K g})$ & $\mathbf{X} \pm \mathbf{D E}$ & Med & $\mathbf{p 1}$ & $\mathrm{p} 2$ \\
\hline Control negativo & - & $0,17 \pm 0,17$ & 0 & 0,004 & - \\
Control positivo & - & $3,50 \pm 1,06$ & 2,5 & - & 0,004 \\
IND/etanol + extracto inca muña & 50 & $2,83 \pm 0,80$ & 2,5 & 0,684 & 0,004 \\
IND/etanol + extracto inca muña & 150 & $1,17 \pm 0,54$ & 1 & 0,084 & 0,152 \\
IND/etanol + extracto inca muña & 300 & $0,50 \pm 0,34$ & 0 & 0,011 & 0,461 \\
IND/etanol + ranitidina & 50 & $0,83 \pm 0,41$ & 0,5 & 0,026 & 0,180 \\
\hline
\end{tabular}

* Los datos de ninguno de los extractos presentó distribución normal $(p<0,05$ Kolmogorov-Simirnov) $\mathbf{X}$ : media; DE: desviación estándar; Med: mediana; IND: indometacina, p1: comparación entre las medianas de las dosis del extracto y de la indometacina $2,5 \mathrm{mg} / \mathrm{kg}$, (Prueba de Mann-Whitney); p2: comparación entre las medianas de las dosis del extracto y del suero fisiológico, (Prueba de Mann-Whitney).

\section{CONCLUSIÓN}

- El extracto etanólico de Clinopodium bolivianuma dosis de $300 \mathrm{mg} / \mathrm{kg}$ presentó mejor efecto gastroprotector, evidenciándose a nivel macroscópico y microscópico sobre las lesiones gástricas inducidas en ratones. 
Asimismo se identificó la presencia de compuestos fenólicos, flavonoides, triterpenos y alcaloides.

\section{REFERENCIAS BIBLIOGRÁFICAS}

1. Alqasoumi S, Al-Yahya M, Al-Howiriny TA, Rafatullah S. Gastroprotective effects of radish Raphanus sativus $\mathrm{L}$. on experimental gastric ulcer models in rats. Farmacia. 2008; 56(2): 204-14.

2. Bandyopadhyay D, Biswas K, Bhattacharyya M, Reiter RJ, Banerjee RK. Involvement reactive oxygen species in gastric ulceration: protection by melatonin. Indian J Exp Biol. 2002; 40(6):693-705.

3. Boligon AA, De Brum TF, Frolhich JK, Froeder ALF, Athayde ML. HPLC/DAD profile and determination of total phenolics, flavonoids, tannins and alkaloids contents of Scutia buxifolia Reissek stem bark. Res J Phytochem. 2012; 6(3): 84-91.

4. Agapito T, Sung I. Fitomedicina: 1100 plantas medicinales. Lima: Edit. Isabel, 2002.

5. Brack, A. Diccionario enciclopédico de plantas útiles del Perú. Cusco: CBC-Centro de Estudios Regionales Andinos «Bartolomé de las Casas», 1999.

6. Senatore F. Volatile constituents of Minthostachys setosa (Briq.) Epl. (Lamiaceae) from Peru. Flavour and Fragrance Journal, 1998; 13(4): 263-65.

7. Lock O. Investigación Fitoquímica. Métodos en el Estudio de Productos Naturales. $2^{\mathrm{da}}$ edición. Lima: Fondo Editorial Pontificia Universidad Católica del Perú, 1994. p. 33-5.

8. Robert A, Nezamis JE, Lancaster C, Davis JP, Field SO, Manchar AJ. Mild irritants prevented gastric necrosis through 'adaptive cytoprotection' mediated by prostaglandins. Am J Physiol. 1983, 245(1): G113-G21.

9. Halliwell B, Gutteridge A. Free Radicals in Biology and Medicine. UK: Clarendon Press Oxford, 1987. p. 247.

10. Süzgeç-Selçuk S, Birteksöz AS. Flavonoids of Helichrysum chasmolycicum and its antioxidant and antimicrobial activities. S Afr J Bot. 2011, 77(1): 170-4.

11. Laine L, Takeuchi K, Tarnawski A. Gastric mucosal defense and cytoprotection: bench to bed side. Gastroenterology. 2008; 135(1): 41-60.

12. Shaker E, Mahmoud H, Mnaa S. Anti-inflammatory and anti-ulcer activity of the extract from Alhagi maurorum (camelthorn). Food Chem Toxicol. 2010; 48(10): 2785-90.

13. Vonkeman HE, Klok RM, Postma MJ, Brouwers JR, Van de Laar MA. Direct medical costs of serious gastrointestinal ulcers among users of NSAIDs. Drugs Aging. 2007; 24(8): 681-90.

14. Ineu RP, Pereira ME, Aschner M, Nogueira CW, Zeni G, Rocha JB. Diphenyl diselenide reverses gastric lesions in rats: involvement of oxidative stress. Food ChemToxicol. 2008; 46(9): 3023-9.

15. Sowndhararajan K, Kang SC. Protective effect of ethyl acetate fraction of Acacia ferruginea DC. Against ethanol-induced gastric ulcer in rats. J Ethnopharmacol. 2013; 148(1): 175-81.

16. Cadirci E, Suleyman H, Aksoy H, Halici Z, Ozgen U, Koc $\mathrm{A}$, et al. Effects of Onosma armeniacum root extract on ethanol-induced oxidative stress in stomach tissue of rats. Chem Biol Interact. 2007; 170(1): 40-8.

17. Mota CS, Freitas RB, Athayde ML, Boligon AA, Augusti P, Somacal S, et al. Effect of Vernonia cognata on oxidative damage induced by ethanol in rats. Hum Exp Toxicol. 2011; 30(7): 675-84.

Manuscrito recibido el: 22/05/2015

Aceptado para su publicación el: 02/11/2015

\section{Correspondencia:}

Nombre: $\quad$ Oscar Herrera Calderón

Dirección: Universidad Nacional San Luis Gonzaga; Campus Universitario (Panamericana Sur Km 305 - Ica)

e-mail: $\quad$ oh2ooo_4@hotmail.com 